\title{
cAMP signalling is involved in growth, germination, mycoparasitism and secondary metabolism in Trichoderma virens
}

\author{
Correspondence \\ Prasun K. Mukherjee \\ prasunmukherjee1@gmail.com
}

Received 3 January 2007

Revised 12 February 2007

Accepted 14 February 2007

\author{
Mala Mukherjee, Prasun K. Mukherjee and Sharad P. Kale \\ Nuclear Agriculture and Biotechnology Division, Bhabha Atomic Research Centre, \\ Mumbai 400 085, India
}

\begin{abstract}
An adenylate-cyclase-encoding gene, tac1, of Trichoderma virens, a soil fungus used in the biocontrol of plant pathogens, has been cloned and sequenced. The tac1 ORF spanned $7032 \mathrm{bp}$, encoding a protein of 2153 aa, which shared an identity of $65 \%$ with the adenylate cyclase of Colletotrichum lagenarium. Deletion of tac1, through double-crossover homologous recombination, lowered the intracellular cAMP levels to below the detection limit. The mutants showed only 5-6\% of the wild-type growth rate on agar, but grew normally in shake culture. The mutants did not sporulate in darkness, and the spores failed to germinate in water. In the confrontation assay, the mutants did not overgrow the test plant pathogens Sclerotium rolfsii, Rhizoctonia solani and Pythium sp. Against Pythium sp., the mutants produced a clear zone of inhibition in the confrontation assay. HPLC analysis and bioassay showed reduced secondary metabolite production in the mutants. Using suppression subtractive hybridization (SSH), the genes that were underexpressed in the mutants were identified. Based on an array of $53 \mathrm{SSH}$ library clones, 11 clones were identified as strongly downregulated in the $\Delta t a c 1$ mutants; of these 11 clones, nine sequences were homologous to secondary metabolism-related gene sequences. Therefore, cAMP signalling positively regulates secondary metabolism in $T$. virens. This is believed to be the first direct genetic study on the role of cAMP signalling in a Trichoderma sp. Tac1 is also believed to be the first regulatory protein to be identified in $T$. virens that is involved in growth, germination, mycoparasitism and secondary metabolism.
\end{abstract}

\section{INTRODUCTION}

cAMP, produced from ATP by adenylate cyclase, regulates many of the biological activities of prokaryotic and eukaryotic cells (Pastan \& Perlman, 1970; Robinson \& Sutherland, 1971). Although cAMP is an important regulator of growth, development and pathogenicity in filamentous fungi, genes encoding adenylate cyclase have been studied in a few species only (Adachi \& Hamer, 1998; Choi \& Dean, 1997; Fillinger et al., 2002; Ivey et al., 2002; Klimpel et al., 2002; Liebmann et al., 2003). The crisp mutants of Neurospora crassa which lack a functional copy of the adenylate cyclase are characterized by a colonial growth habit, with impairment in elongation of aerial hyphae, and a premature accumulation of conidia (Rosenberg \& Pall, 1979; Terenzi et al., 1974, 1976). Evidence from the analysis of Aspergillus nidulans strains defective for adenylate cyclase (CyaA) or PkaA has shown the involvement of cAMP signalling in conidial germination in response to carbon-source sensing (Fillinger et al., 2002).

Abbreviation: $\mathrm{SSH}$, suppression subtractive hybridization.

The GenBank/EMBL/DDBJ accession no. for the sequence reported in this paper is EF189190.
In PkaA-defective strains, cAMP levels have been shown to increase dramatically by 250 -fold, indicating that PkaA regulates intracellular cAMP levels (Fillinger et al., 2002). In the human pathogen Aspergillus fumigatus, disruption of the adenylate-cyclase-encoding gene acyA results in reduced colony diameter and conidiation; the mutants are less virulent as a result of reduced defence against killing by macrophages (Liebmann et al., 2003). In the rice pathogen Magnaporthe grisea, macl disruption results in abolition of appressorium formation, and reduction in vegetative growth, conidiation and conidial germination (Adachi \& Hamer, 1998; Choi \& Dean, 1997). $\Delta$ magB (Gprotein) and $\Delta$ macl (adenylate cyclase) mutants share several phenotypes, indicating that adenylate cyclase is downstream of MagB in M. grisea (Liu \& Dean, 1997). The $\mathrm{G}_{\beta}$ subunit MGB1 is involved in cAMP signalling for regulating conidiation, surface recognition and appressorium formation in M. grisea (Nishimura et al., 2003). In Botrytis cinerea, the adenylate cyclase (BAC) is required for full pathogenicity (Klimpel et al., 2002).

Trichoderma spp. are economically important as sources of many industrial enzymes, and as commercial biofungicides (Harman \& Bjorkmann, 1998; Hjeljord \& Tronsmo, 1998; 
Mach \& Zeilinger, 2003). These fungi inhabit soil and the rhizosphere, and inhibit other fungi through antibiosis and mycoparasitism (Chet et al., 1998; Sivasithamparam \& Ghisalberti, 1998). They are also good plant growth promoters, and they induce resistance in plants against invading pathogens through limited colonization of plant roots (Harman et al., 2004; Viterbo et al., 2005). Two species (Trichoderma longibrachiatum and Trichoderma citrinoviride) are opportunistic human pathogens (Kuhls et al., 1999). Trichoderma virens IMI 304061 is an aggressive mycoparasite on the plant pathogens Sclerotium rolfsii and Rhizoctonia solani (Mukherjee et al., 1995). To understand the role of signalling pathways in mycoparasitism and development, we previously isolated loss-of-function mutants for two $\mathrm{G}_{\alpha}$ proteins (TgaA and TgaB) and an MAPK (TmkA) (Mukherjee et al., 2003b; 2004). However, there has been no direct evidence reported in the literature for a role of cAMP signalling in any Trichoderma species. Antisensemediated silencing of the $\mathrm{G}_{\alpha}$ protein Tgal results in reduced cAMP level in Trichoderma atroviride IMI 206040 (RochaRamirez et al., 2002). The mutants are hypersporulating, and have reduced mycoparasitic coiling. Interestingly, deletion of Tga1 in T. atroviride ATCC 78058 results in elevated internal cAMP levels (Reithner et al., 2005). These mutants also show hypersporulation, a total loss of mycoparasitism, and increased antibiosis against $R$. solani, even though the production of an antifungal metabolite, 6-pentyl- $\alpha$-pyrone, is reduced. The deletion of another $\mathrm{G}_{\alpha}$ protein, Tga3, results in reduced intracellular cAMP levels in T. atroviride, and is associated with reduced growth rate and germination, lightindependent conidiation, and loss of mycoparasitism (Zeilinger et al., 2005). It is interesting that the phenotypes associated with the deletion of Tgal or Tga3 are similar, although the cAMP level is elevated in Tgal mutants and reduced in Tga3 mutants. Recently, Casas-Flores et al. (2006) studied the role of a protein kinase A regulatory subunit in blue-light-induced conidiation of T. atroviride. Expression of an antisense copy of this gene results in a non-sporulating phenotype, whereas overexpression results in light-independent conidiation, indicating the involvement of cAMP signalling in conidiation in T. atroviride. In contrast to most of the fungi studied, including the closely related $T$. atroviride, the deletion of the $\mathrm{G}_{\alpha}$ protein TgaA has no effect on growth, sporulation or spore germination frequency in $T$. virens (Mukherjee et al., 2004). This raised the question of whether the functioning of the G-protein-cAMP pathway in T. virens differs from other ascomycetes. Also, what are the physiological roles that this pathway plays in this fungus? Since there has been no direct evidence on the role of cAMP signalling through the deletion of adenylate cyclase in any Trichoderma sp., we cloned the adenylate-cyclase-encoding gene $t a c 1$ of $T$. virens, which is an important biocontrol fungus, and studied the role of the cAMP signalling by gene knockout.

\section{METHODS}

Fungal strains and growth conditions. T. virens (IMI 304061), S. rolfsii (MTCC 6052), R. solani (ITCC 4110) and Pythium sp. have been used in our previous studies (Mukherjee et al., 2006a). Routinely, the fungi were grown on potato glucose agar (PDA; Difco) at ambient temperature $\left(26-28{ }^{\circ} \mathrm{C}\right)$, and stored as glycerol stocks at $-80{ }^{\circ} \mathrm{C}$ for long-term storage and maintenance of genetic stability. For growth on dialysis membranes, $10^{6}$ conidia were spread on PDA plates overlaid with a sterile dialysis membrane (MWCO 12 000-14000; Spectra/Por), and incubated for 2 days at ambient temperature. The mycelial mat was harvested with a sterile spatula, frozen in liquid nitrogen, and stored at $-80{ }^{\circ} \mathrm{C}$ until further use. For analysis of the secondary metabolites, the agar was extracted in solvent as described below.

Cloning of the full-length gene. Using the M. grisea macl sequence, two primers, ACFor (CCATATGACTGGAATTGGAA) and ACRev (ACCATGAAGGCGTCACCT), were designed from the region showing high homology with other fungal adenylate cyclase gene sequences, and part of the gene $(1.4 \mathrm{~kb})$ was amplified from $T$. virens genomic DNA by PCR. This fragment was cloned, and used as a probe for screening a $T$. virens cosmid library described previously (Mukherjee et al., 2003b). The full-length gene was subcloned from the cosmid clone by a three-step inverse PCR using the primer pairs INV1 (GCCGCCTCATGCTGTCTG) and INV2 (GGAGATGTCCTCGACTCTTC), INV2 and INV3 (GAATATGCGAATAAAGTAAGG), and INV4 (GTTCGGGAGGCTGCTCTCG) and INV5 (GCCTGCGATCCACGAATAG). The 3' end of the gene was cloned as a DraI fragment amplified with the gene-specific primer Xin1.22GW1 (GATCGGAAGCCTCTTGCGAAGATGAG) by using the Clontech GenomeWalker Universal kit (BD Biosciences). All the clones were sequenced, assembled and translated by using Gene Runner software, and the putative ORF and introns were identified. Homology with adenylate cyclase from other fungi was studied by BLASTP on the NCBI server.

Construction of the gene-deletion construct, protoplast transformation, and selection and purification of transformants. A double-crossover construct, pACDC, was made by replacing $5087 \mathrm{bp}$ of the ORF with a hygromycin-resistance cassette consisting of $h p h$ (hygromycin phosphotransferase), TrpC promoter and TrpC terminator, taken from the plasmid pAT-BS (Mukherjee et al. 2003a). The linear construct (Fig. 1a), consisting of the left flank (2441 bp), the marker $(2.2 \mathrm{~kb})$, and the right flank (2010 bp), was amplified by the primer pair AC5'2s (GAGATGCGAGACTGTCAGCG) and ACRFNot (CAATGATATGAGAGCGGCCGCCATTC), and was used for transforming $T$. virens protoplasts, as described previously (Mukherjee et al., 2003b). The transformants were selected using $200 \mathrm{mg}$ hygromycin $\mathrm{B}^{-1}$ (Roche), and transferred to PDA plates containing $100 \mathrm{mg}$ hygromycin $\mathrm{B}^{-1}$. The transformants that showed non-wild-type phenotypes were purified by repeated single-spore isolation, and three putative knockout mutants were selected based on atypical colony growth and morphology.

Southern hybridization, PCR and RT-PCR. High-molecular-mass genomic DNA from the wild-type (WT) and the candidate $\Delta$ tacl mutants (hereafter referred to as 'mutants') was extracted, and subjected to PCR and Southern analysis. PCR was performed by the primer pair INV3 and INV5 for the adenylate cyclase gene, and hphfor (GAGGGCGAAGAATCTCGTGC) and hphrev (CACTGACGGTGTCGTCCATC) for the $h p h$ gene. For genomic Southern analysis, the DNA was digested with $\mathrm{XbaI}$, and hybridized with a $1.1 \mathrm{~kb}$ fragment amplified from the genomic DNA using the primer pair INV3 and INV5. For analysis of the tac1 transcript level, total RNA was isolated from the mycelial mat of the WT and mutants grown on the dialysis membrane, and first-strand cDNA was synthesized from $1 \mu \mathrm{g}$ total RNA using the First Strand cDNA Synthesis kit (Roche) in a $20 \mu \mathrm{l}$ reaction volume. A $5 \mu \mathrm{l}$ volume of the first-strand cDNA was used for amplification with Taq polymerase (Genei) in a $25 \mu$ l volume, using the primer pairs INV3 and INV5 (for 
tac1), hphfor and hphrev (for $h p h$ ), and h3for (GCCCGCACCAAGCAGACCG) and h3rev (GGCGGGCGAGCTGGATGTC) (for histone3).

Assay for intracellular cAMP concentration. For measuring the intracellular cAMP concentration, the WT strain and the mutants were grown on a dialysis membrane, as described above. Two-day-old mycelial mat was harvested, frozen in liquid nitrogen, ground, homogenized in 10 vols $0.1 \mathrm{M} \mathrm{HCl}$, and centrifuged $(600 \mathrm{~g})$. The cAMP level was measured by using the Direct cAMP Enzyme Immunoassay kit (Sigma), as per the manufacturer's instructions. Protein concentration was determined by using the Folin phenol reagent (Lowry method). The intracellular cAMP concentration was expressed as pmol (mg protein $)^{-1}$.

Growth and morphology of the mutants. Mycelial discs were inoculated on PDA plates, and the linear growth measured. For studying the colony development from single spores, PDA plates were spread with diluted conidial suspension. Dry weight (biomass) was measured after 3 days of shake incubation of $100 \mathrm{ml}$ potato glucose broth $(\mathrm{PDB})$ inoculated with $10^{5}$ conidia. For the germination assay, conidia were harvested from 10-day-old cultures grown on PDA plates, and washed thrice in double-distilled water. Approximately $5 \times 10^{4}$ conidia in $50 \mu \mathrm{l}$ water were incubated on sterile glass slides in a moist chamber. The conidia were observed for germination after $20 \mathrm{~h}$.

Confrontation assay. The ability of the mutants and the WT to overgrow and lyse the mycelia of the test plant pathogens was assessed using a confrontation assay on PDA plates (Mukherjee et al., 2003b). Observation was recorded for overgrowth of Trichoderma on the test fungi, and for the lysis of the mycelia.

Antibiosis and HPLC analysis. The ability of the mutants and the WT to inhibit the growth of Pythium sp. through the production of diffusible antibiotics was assessed by bioassay and HPLC. Conidial suspension was spread on dialysis membrane, and grown for 2 days, as described above. The dialysis membrane and the mycelial mat were removed, and the agar was blended in $80 \%$ aqueous acetone (1 g agar in $3 \mathrm{ml}$ acetone), and centrifuged at 10000 r.p.m. (13000 g) for $10 \mathrm{~min}$. The supernatant was extracted with an equal volume of chloroform. The chloroform was evaporated under $\mathrm{N}_{2}$ flush, and the metabolites were reconstituted in methanol (1/100 original volume). A $10 \mu \mathrm{l}$ volume of the reconstituted metabolites was assayed by the agar-well technique, in which the solution was added to a $5 \mathrm{~mm}$ well that had been cut at the centre of a PDA plate. After evaporation of the solvent, three mycelial discs of Pythium sp. were inoculated near the edge of the plate, and photographed after 2 days incubation. HPLC was performed as described previously (Mukherjee et al. 2006a).

Suppression subtractive hybridization (SSH), array and sequencing of the differential clones. Total RNA was isolated from the mycelial mat of the WT and the $\Delta t a c 1$ mutant ACM1 grown on a dialysis membrane, as described above. First-strand cDNA from the WT and ACM1 total RNA was synthesized using the BD SMART RACE cDNA amplification kit with 3 '-RACE CDS Primer A and BD SMART IIA oligonucleotide (BD Biosciences). The first-strand cDNA was purified by selective precipitation (ammonium acetate precipitation), and the second-strand cDNA was amplified using $2.5 \mu \mathrm{l}$ firststrand cDNA as the template, the Nested Universal Primer A (BD Biosciences) as the oligonucleotide, and Advantage polymerase (BD Biosciences), in a reaction volume of $100 \mu$ l. The cycling parameters were as follows: initial denaturation at $95{ }^{\circ} \mathrm{C}$ for $1 \mathrm{~min}$, then 17 cycles (optimized initially by taking samples up to 30 cycles, based on the protocol described in BD Super SMART-PCR cDNA synthesis kit) at $95{ }^{\circ} \mathrm{C}$ for $15 \mathrm{~s}, 65{ }^{\circ} \mathrm{C}$ for $30 \mathrm{~s}$, and $68{ }^{\circ} \mathrm{C}$ for $6 \mathrm{~min}$. The amplified
cDNA was treated with RNaseA (Roche), purified using the High Pure PCR product purification kit (Roche), reconstituted in $20 \mu \mathrm{l}$ nuclease-free water, and the concentration was determined spectrophotometrically. Amplified cDNA $(2 \mu \mathrm{g})$ from the WT or the $\Delta t a c 1$ mutant ACM1 was subjected to SSH using the Clontech PCR-Select cDNA subtraction kit (BD Biosciences), according to the manufacturer's instructions. The WT cDNA was used as the tester, and the mutant cDNA was used as the driver. After the second (nested) PCR, the products were cloned in the pTZ57R/T vector (Fermentas). The presence of single inserts was confirmed by colony PCR using the Nested PCR primers 1 and 2R, which were supplied with the kit, and 53 clones showing a single insert were arrayed on a positively charged nylon membrane (Roche). Histone $h 3$ cDNA was included as a control. A $2 \mu \mathrm{l}$ volume of the heat-denatured PCR product of each clone was blotted on membranes, and hybridized with WT or mutant cDNA, which was labelled with $\left.{ }^{32} \mathrm{P}\right] \mathrm{dCTP}$ (BRIT). After hybridization, the blots were exposed to X-ray films overnight. The clones that were clearly differential were purified, then sequenced with T7 primer using an automated sequencer, and homologous sequences in the database were identified by BLASTX on the NCBI server.

\section{RESULTS}

\section{Isolation of tac1}

Using the primer pair ACFor and ACRev, taken from the $M$. grisea macl sequence in the region of greatest similarity to other fungal adenylate cyclase genes, a $1.4 \mathrm{~kb}$ fragment was cloned and sequenced. BLASTX analysis confirmed the fragment to be part of the adenylate cyclase gene. Using this product as the probe, a cosmid library was screened, and a cosmid clone of approximately $26 \mathrm{~kb}$ was confirmed to harbour the adenylate cyclase gene by PCR and Southern hybridization (data not presented). Using inverse PCR, a fragment of approximately $7.3 \mathrm{~kb}$ was cloned in three steps, and the missing $3^{\prime}$ end was cloned by genome walking as a $800 \mathrm{bp}$ DraI fragment. Together, we cloned a $9623 \mathrm{bp}$ sequence containing the entire coding region (7032 bp), the upstream region (2523 bp), and the $68 \mathrm{bp}$ sequences downstream of the stop codon; the entire 9623 bp sequence has been deposited under GenBank accession no. EF189190. The ORF, encoding a protein of 2153 aa, is interrupted by three introns of 174, 327 and $69 \mathrm{bp}$. The $T$. virens adenylate cyclase Tacl is highly homologous to adenylate cyclases from other fungi, such as Colletotrichum lagenarium, M. grisea and Podospora anserina (65, 62 and $59 \%$ identity, respectively, at the amino acid level).

\section{Isolation of Tac1 loss-of-function mutants}

Using protoplast-mediated transformation of $T$. virens, with the disruption cassette amplified from the plasmid construct pACDC, several hundred transformants were obtained by selection on $200 \mathrm{mg}$ hygromycin $\mathrm{B} \mathrm{l}^{-1}$. One hundred transformants were transferred to fresh PDA containing $100 \mathrm{mg}$ hygromycin $\mathrm{B}^{-1}$, and three colonies were found to be extremely slow growing, and they did not appear to be similar to the WT colony. These three colonies were purified by repeated single-spore isolations. The 
mutants (designated ACM1, ACM2 and ACM3) were confirmed for deletion of the adenylate cyclase gene by genomic PCR, Southern hybridization and RT-PCR. Using the primer pair INV3 and INV5, we obtained a $1.1 \mathrm{~kb}$ band in the WT, and this was found to be absent in the three mutants (Fig. 1b). All the mutants were positive for the $h p h$ gene, which was not detected in the WT (Fig. 1b). Gene deletion was also confirmed by Southern hybridization (data not presented). RT-PCR data (Fig. 1c) indicated the absence of the tacl transcript in the mutants, but it was present in the WT. The opposite was true for the $h p h$ transcript. The intracellular cAMP level in the 2-day-old WT mycelial mat harvested from the dialysis membrane overlying PDA was $6.227 \pm 0.415 \mathrm{pmol}$ (mg protein $)^{-1}$. The cAMP levels in the mutants were below the detection limit, although the protein level ranged from 1.19 to $1.57 \mu \mathrm{g}$ $(\mu \mathrm{l} \text { extract })^{-1}$, compared with $0.59 \mu \mathrm{g}(\mu \mathrm{l} \text { extract })^{-1}$ in the WT.

\section{Growth, morphology, and conidial germination}

The $\Delta$ tac1 mutants, when grown on PDA plates from the disc inoculum, showed a marked reduction in growth rate $\left(1.1 \pm 0.3 \mathrm{~mm} \mathrm{day}^{-1}\right.$ linear growth, compared with WT growth of $19.2 \pm 1.4 \mathrm{~mm}$ day $\left.^{-1}\right)$. The WT fully covered the $85 \mathrm{~mm}$ plates in 2 days, while the mutants took more than a month to cover the entire plate (Fig. 2a). The mutants had aerial growth, and sporulated initially towards the centre of the plates, but subsequently they showed submerged growth devoid of aerial hyphae and conidiation. A dark-yellow pigment diffusing from the mutant colonies was seen, and the mutant colony growth was very compact. When PDA plates with the WT or mutants were incubated in the dark, the mutants failed to form conidia, but the colonies responded to light by forming green conidia when incubated under constant illumination for 2 days (Fig. 2b). When the plates were inoculated with a diluted conidial suspension, the mutant colonies were visible 2 days after plating, as opposed to the WT colonies, which were visible after 1 day. The mutant colonies from single spores were very small, discrete and compact, and they produced mainly aerial hyphae, in contrast to the typical spreading growth habit of the WT strain (Fig. 2c). On subsequent incubation, the mutant colonies began to sporulate, produce dark-yellow diffusible pigments, and they showed a submerged growth habit. In 3-day-old liquid shake culture, there was no significant difference between the WT and the mutants with respect to dry matter production $(672 \pm 21 \mathrm{mg}$ for the WT, compared with $630 \pm 49,640 \pm 40$ and $657 \pm 31 \mathrm{mg}$ for the mutants ACM1, ACM2 and ACM3, respectively). The mutants, when transferred from liquid culture to agar medium, grew typically as slow-growing mutant colonies. This shows that no irreversible modification occurred during growth in

(a)

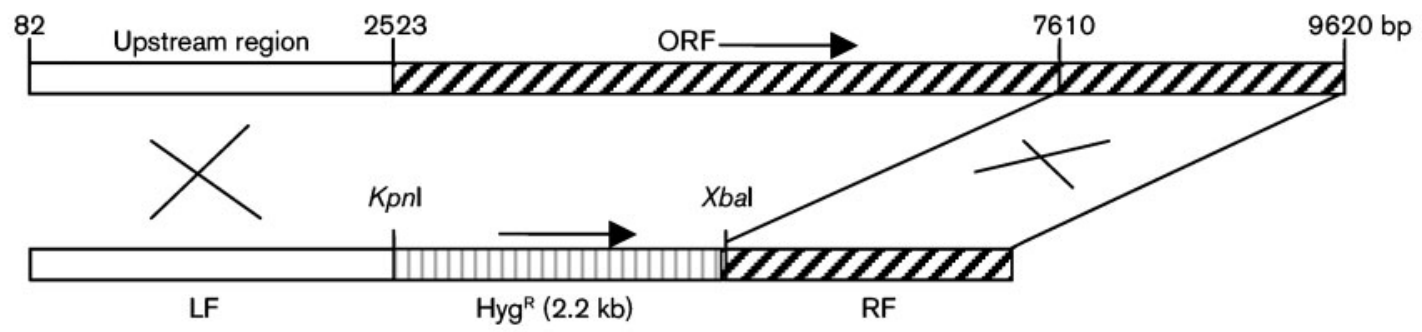

(b)

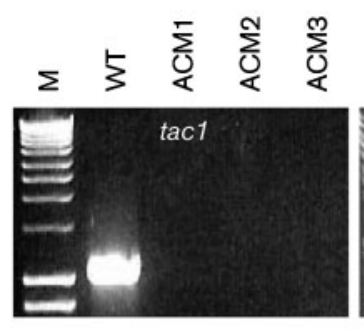

(c)

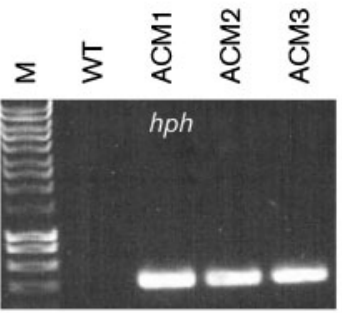

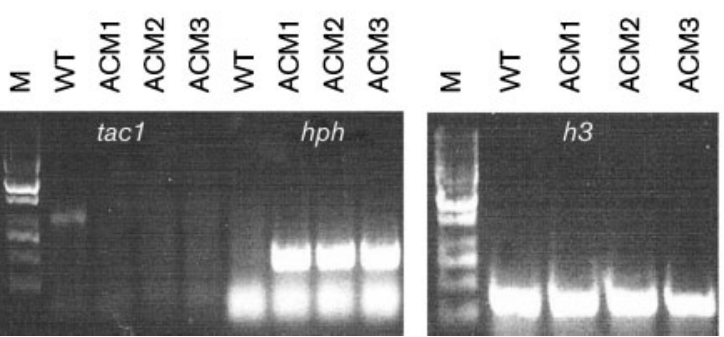

Fig. 1. Disruption of tac 1 by homologous recombination. (a) Strategy for double-crossover homologous integration: a 5087 bp section of the ORF was replaced with a $2.2 \mathrm{~kb}$ hygromycin-resistance cassette $\left(\mathrm{Hyg}^{\mathrm{R}}\right)$, resulting in a construct with a 2441 bp upstream region as the left flank (LF), and a 2010 bp right flank (RF). (b) PCR amplification of tac1 and $h p h$ from the genome of the WT and $\Delta \operatorname{tac} 1$ mutants (ACM1, ACM2 and ACM3). (c) RT-PCR analysis showing the presence of the tac1 transcript in the WT, and the absence of the tac1 transcript in the mutants, and the presence of the $h p h$ transcript in the mutants, but not in the WT. Histone $h 3$ was included as a loading control. M, molecular-mass marker (hyperladder, 0.2-10 kb). 
(a)

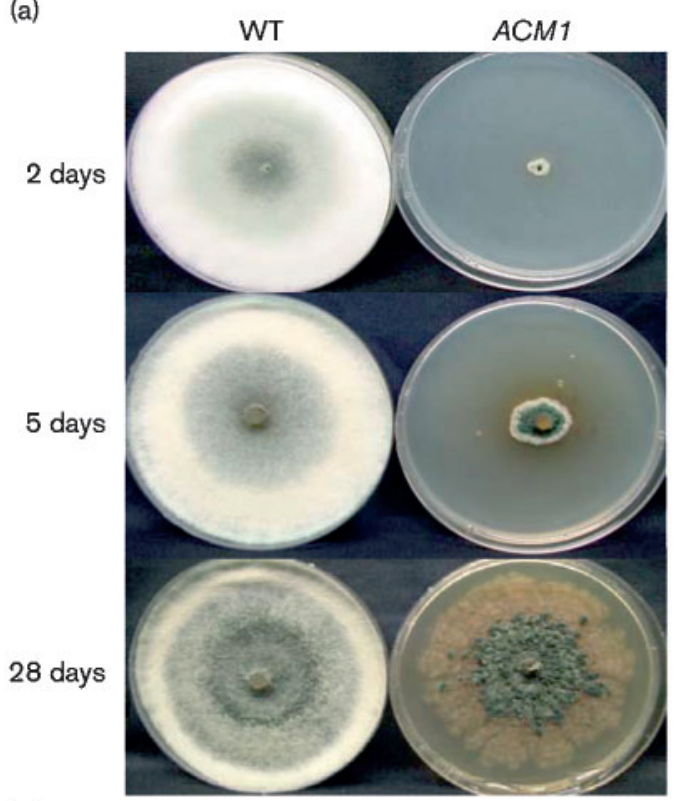

(c)

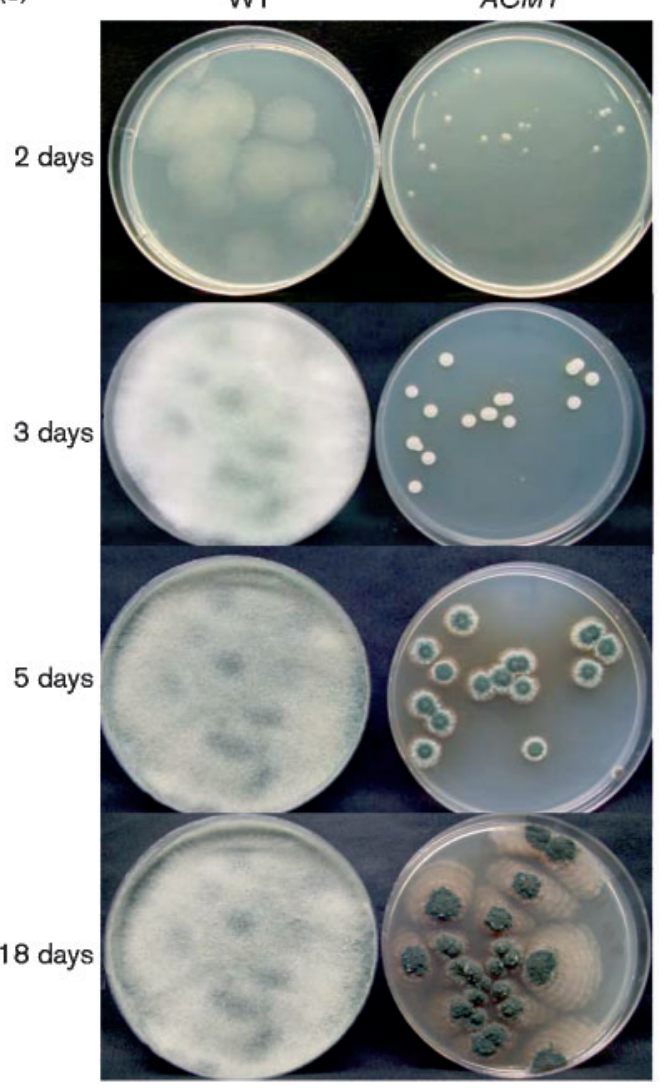

(b)

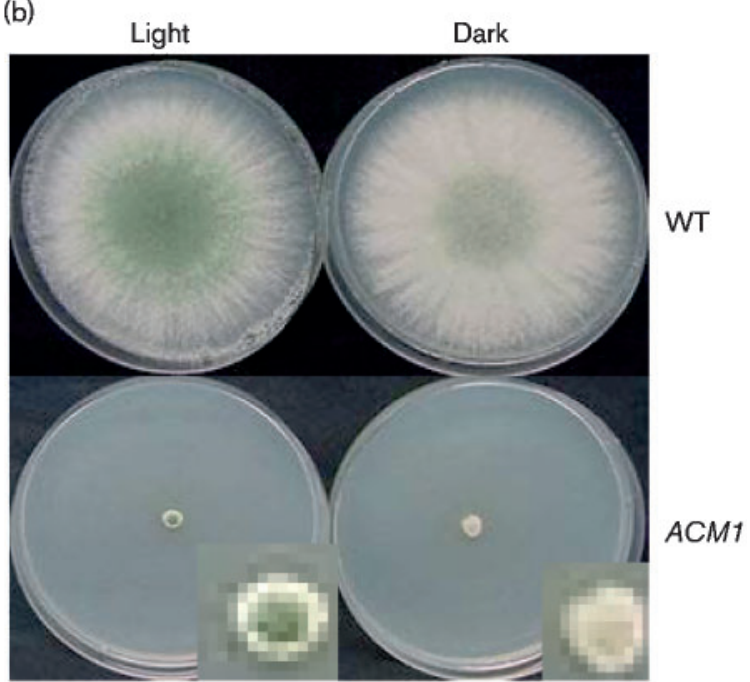

(d)

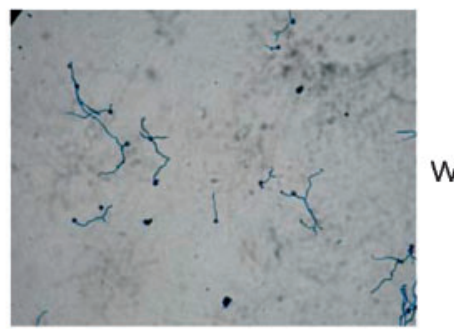

WT

ACM1

Fig. 2. Growth and morphology of $T$. virens $W T$ and the $\Delta \operatorname{tac} 1$ mutant ACM1. (a) Growth on PDA plates from a disc inoculum after 2, 5 and 28 days incubation. (b) Growth and conidiation of the WT and the $\Delta$ tac 1 mutant on PDA from disc inoculum after 2 days incubation in the dark and in continuous light. Inset, magnified view of the mutant colonies to show the presence of conidiation in the light, and absence of conidiation in the dark. Note, some conidiation in the WT incubated in the dark was due to nutrient stress as the colony reached the margin of the plate. (c) Growth of the WT and the $\Delta \operatorname{tac} 1$ mutant on PDA plates seeded with conidia after 2, 3, 5 and 18 days incubation. (d) Germination of conidia of the WT and $\Delta$ tac 1 mutant in water after $20 \mathrm{~h}$ incubation. 
liquid medium. When incubated in water for $20 \mathrm{~h}$, the conidia from the mutants failed to germinate, while nearly all the conidia of the WT strain germinated through the production of normal germ tubes (Fig. 2d).

\section{Confrontation assay, and secondary metabolite production}

In confrontation assays, where the test plant pathogens were inoculated opposite the Trichoderma strains, the WT and the test plant pathogens met around the centre of the plate, and, with time, the WT started to overgrow the colonies of the test fungi. The WT fully overgrew and lysed the colonies R. solani, S. rolfsii and Pythium sp. in 5, 10 and 4 days respectively (Fig. 3a). The mutants, however, failed to overgrow any of the test pathogens, even after prolonged incubation. In contrast, $R$. solani and S. rolfsii overgrew the mutant colonies, indicating that the mutants could not offer resistance to overgrowth by these plant pathogens. Against Pythium sp., a clear zone of inhibition was seen when it was inoculated opposite the mutants. In the bioassay of chloroform-extracted metabolites on Pythium sp., the mutants showed reduced inhibition compared with the WT (Fig. 3b). HPLC analysis of the non-polar fractions showed the presence of several unknown metabolites, along with viridiol, which is a reduction product of viridin. The mutants produced lesser concentrations of metabolites in agar, compared with the WT (Fig. 3c).

\section{SSH identification of genes underexpressed in the mutants}

Using SSH, we made a library of several hundred clones that were thought to represent genes that were underexpressed in the mutants, since WT cDNA was used as the tester, and mutant cDNA was used as the driver. Of the 53 clones arrayed, 11 (clone nos 2, 4, 9, 10, 12, 19, 21, 25, 40, 41 and 44) were strongly differential (Fig. 4); of these, the most abundant was a cytochrome P450 gene (vir2), representing four clones (clone nos 4, 10, 21 and 25). The other clones related to secondary metabolism were cyp1 (a cytochrome P450 gene, clone no. 9), vir4 (a cyclase gene, clone no. 12), vir3 (a cytochrome $\mathrm{P} 450$ gene, clone no. 40), omtB-2 (an $O$-methyl transferase gene, clone no. 41), and a homologue of the GA14-synthase gene of Fusarium proliferatum (a cytochrome $\mathrm{P} 450$ gene, clone no. 44). Clone no. 2 was a homologue of the stress-response gene ric- 1 of N. crassa, while clone no. 19 had no homology in the database.

\section{DISCUSSION}

G-protein-cAMP and MAPK signalling play central roles in growth, development and secondary metabolism in filamentous fungi (Lengeler et al., 2000; Calvo et al., 2002; $\mathrm{Xu}, 2000)$. We have previously established the role of a $T$. virens MAPK TmkA in repression of conidiation in the (a)

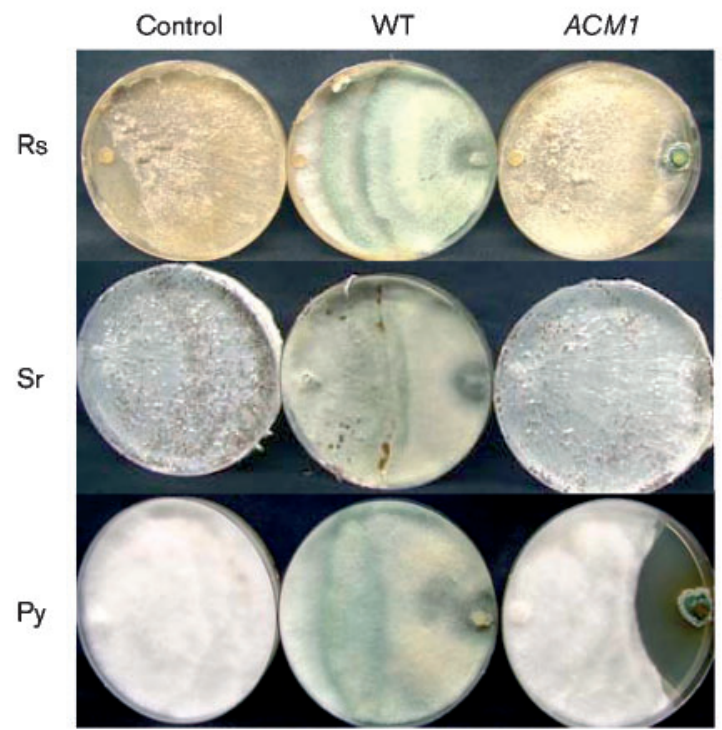

(b)

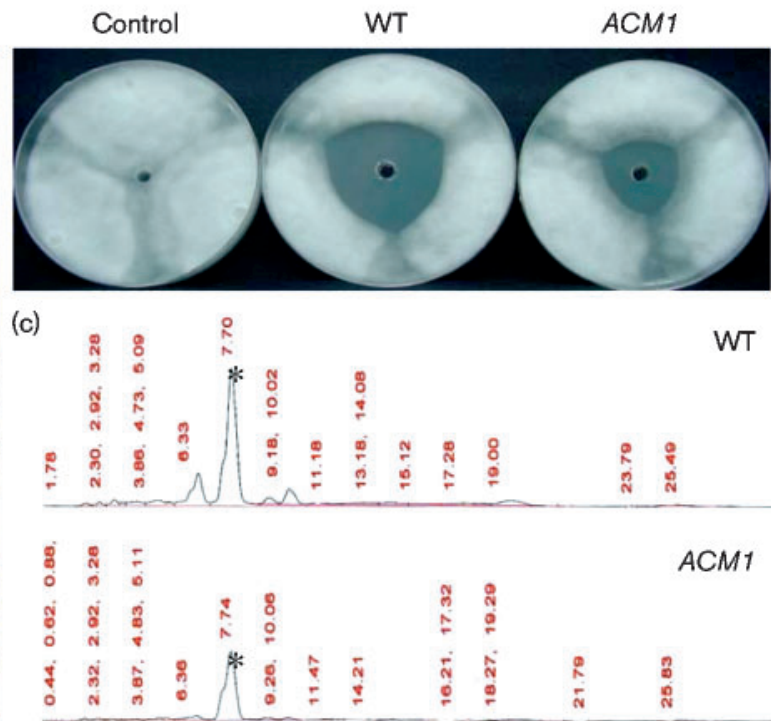

Fig. 3. Antagonism of $T$. virens WT and $\Delta \operatorname{tac} 1$ mutant (ACM1) on R. solani (Rs), S. rolfsii (Sr) and Pythium sp. (Py). (a) Confrontation assay. Plates (except control plates, which were inoculated with the test plant pathogen only) were co-inoculated with the test pathogen (left margin) and Trichoderma (right margin), and incubated at ambient temperature. Photographs were taken after 5, 10 and 4 days co-inoculation with R. solani, S. rolfsii and Pythium sp., respectively. Note the inability of the mutant to overgrow any of the three plant pathogens, and a clear zone of inhibition against Pythium sp. (b) Inhibition of Pythium growth by chloroform-soluble metabolites of the WT and the $\triangle \operatorname{tac} 1$ mutant ACM1. (c) HPLC profile of the same metabolites; the viridiol peak is marked with an asterisk. 
1

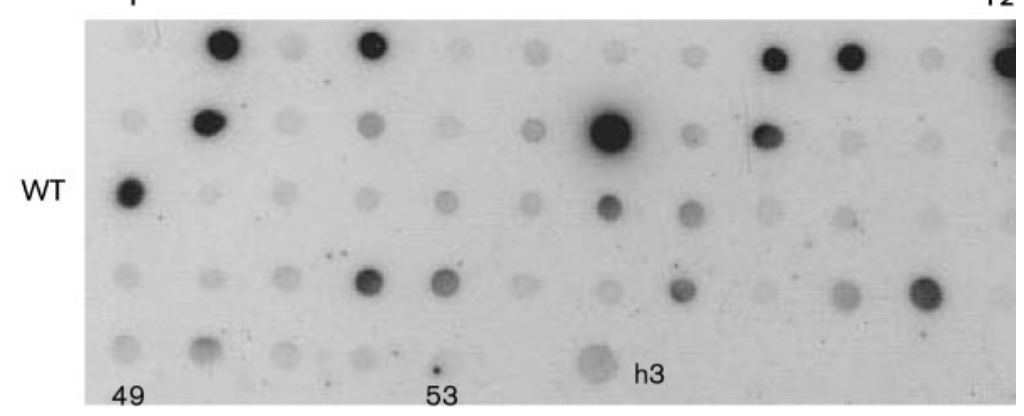

Mutant $1+20+0$ 12

Fig. 4. Array of SSH-derived clones. A total of 53 clones were spotted onto each membrane. h3, histone h3 cDNA. The upper membrane was hybridized with radiolabelled cDNA of the WT, and the lower blot with cDNA from the adenylate cyclase knockout mutant ACM1. Clone nos 2, 4, 9, 10, 12, 19, 21, 25, 40, 41 and 44 were strongly differential, and were analysed further. dark, and identified a gene whose expression is repressed by TmkA (Mukherjee et al., 2003b, 2006b). $\Delta$ tmkA mutants have reduced antagonism against mycelia and sclerotia of some plant pathogens. However, since the deletion of the G-protein TgaA or TgaB does not alter the growth, conidiation or conidial germination frequency of $T$. virens (Mukherjee et al., 2004), the role of cAMP signalling in this fungus has remained elusive. In addition, G-protein-related studies in $T$. atroviride have not established the role of cAMP signalling, since the results have been inconclusive. There is, in general, no direct evidence of cAMP signalling mechanisms in any Trichoderma sp., as, to the best of our knowledge, the gene encoding adenylate cyclase has not been cloned and characterized in any member of this genus. We cloned the $T$. virens gene tac1 encoding adenylate cyclase, and studied its cellular functions. The deletion of the gene through homologous recombination resulted in drastic effects on the colony morphology, and hyphal elongation rate. The linear growth rate was reduced by $94-95 \%$, which is a very strong reduction compared, for example, with $M$. grisea and A. fumigatus, where the deletion of adenylate cyclase reduced the growth rate to 60 and $68 \%$ of the WT, respectively. Though the growth on PDA plates was drastically reduced in the mutants, the growth/biomass production in shake culture was similar to the WT. The normal biomass production in liquid shake culture might be due to a compensatory mechanism through decreased phosphodiesterase activity in submerged culture, as has been suggested for $N$. crassa (Ivey et al., 1999). Alternatively, shake culture may provide a richer environment for growth, as has been suggested for CPG-1 $\left(\mathrm{G}_{\alpha \mathrm{i}}\right)$ mutants of Cryphonectria parasitica (Segers \& Nuss, 2003). Amendment of the medium with $5 \mathrm{mM}$ cAMP did not restore the phenotypes in the mutants (data not presented). The phenotypes of A. fumigatus cyaA mutants and T. atroviride Tga3 mutants, likewise, are not rescued by the addition of cAMP (Liebmann et al., 2003; Zeilinger et al., 2005).

Unlike the G-protein mutants of T. atroviride, and MAP kinase mutants of T. atroviride and T. virens (Mukherjee et al., 2003b, 2006b; Reithner et al., 2005; Zeilinger, 2004; Zeilinger et al., 2005), the $\Delta t a c 1$ mutants did not sporulate constitutively in darkness, and responded to light in the same way as the WT. Thus, the regulation of conidiation in these two closely related species appears to be different: cAMP-dependent regulation in T. atroviride, and cAMPindependent regulation in T. virens. Also, with respect to the regulation of conidiation, there could be a cross-talk between the Tmk1 and cAMP pathways in T. atroviride, but not in T. virens.

In the confrontation assay, $\Delta \operatorname{tac} 1$ mutants were not able to overgrow and lyse the colonies of the test plant pathogens S. rolfsii, $R$. solani and Pythium sp. (Fig. 3a). The loss of virulence against the host fungi could be associated with extremely slow growth rate of the mutants. Against Pythium sp., however, the mutants produced a prominent zone of inhibition. This visible zone of inhibition could be due to the overproduction of antifungal metabolites that diffuse into agar. However, as measured by both bioassay and HPLC (Fig. 3b, c), secondary metabolite production was decreased in the mutants. This is consistent with the results of SSH, where, out of 11 genes that are underexpressed in the mutants, nine are known to be associated with secondary metabolism in T. virens and other fungi (Mukherjee et al., 2006a). T. virens strain IMI 304061 shows abundant production of the antifungal viridin and its reduction product viridiol in culture (Mukherjee et al., 
2006a). HPLC analysis of the non-polar fraction of the metabolites revealed that the concentration of viridiol was lower in the mutants than in the WT. We did not detect viridin in WT or the mutants, probably because of faster conversion of viridin to viridiol in plate culture compared with liquid shake culture. Nevertheless, the presence of viridiol is an indication of early viridin production. Therefore, it seems that the enhanced antifungal properties of the $\Delta t a c 1$ mutants in the confrontation assay are a result of the slow growth of the mutant colonies, rather than of the enhanced production of secondary metabolites. Reducing the growth rate by adding a sublethal dose of fungicides to visualize the zone of inhibition in Trichoderma-fungal interactions is a standard practice. For example, Howell (1987) amended PDA with $0.4 \mu \mathrm{g} \mathrm{ml}^{-1}$ benomyl to visualize the zone of inhibition produced as a result of gliotoxin formation by a strain of $T$. virens against $R$. solani. The $\Delta \operatorname{tga} 1$ mutants of $T$. atroviride produce a lower amount of the antifungal metabolite 6-pentyl- $\alpha$-pyrone, although there is a clear zone of inhibition in the confrontation assay against $R$. solani (Reithner et al., 2005). Those authors attributed this enhanced antibiosis to the possible overproduction of some unidentified low-molecular-mass compounds by the mutants. The relationship between G-protein-cAMP signalling, conidiation and secondary metabolism is well documented in Aspergillus spp., where increased secondary metabolite production is associated with, or the result of, enhanced conidiation (Calvo et al., 2002; Keller et al., 2005). The deletion of the Aspergillus $\mathrm{G}_{\alpha}$ subunit FadA results in premature conidiation, and enhanced sterigmatocystin production (Hicks et al., 1997). The overexpression of PkaA results in reduced sterigmatocystin biosynthesis, while the deletion of this gene results in hypersporulation and aberrant sterigmatocystin production in $A$. nidulans (Shimizu \& Keller, 2001). T. virens $\Delta$ tac1 mutants did not sporulate constitutively, and had reduced secondary metabolite production. This species, therefore, appears to have a novel regulation of conidiation vis-à-vis secondary metabolism that is different from other fungi studied, for example T. atroviride and Aspergillus spp. The present findings clearly establish that cAMP signalling is essential for growth, conidial germination and biocontrol properties, including mycoparasitism and secondary metabolism, in T. virens. We believe that this is the first report on the cloning and functional analysis of a gene encoding adenylate cyclase in any species of Trichoderma. The findings could help to improve the commercial exploitation of Trichoderma spp. for industrial and agricultural applications by manipulating the cAMP-signalling pathway, e.g. by overexpression/constitutive activation of one or more of the components to enhance secondary metabolite production.

\section{ACKNOWLEDGEMENTS}

M. M. thanks the Council of Scientific and Industrial Research (CSIR), New Delhi, India, for a Senior Research Fellowship, and Dr K. B. Sainis, Director, BioMedical Group, and Dr S. F. D'Souza, Head, NA\&BTD, BARC, for providing the opportunity to work at BARC.
Thanks are also due to Mr P. D. Sherkhane for his help with HPLC analysis, and Dr Benjamin A. Horwitz for a critical review of the manuscript.

\section{REFERENCES}

Adachi, K. \& Hamer, J. E. (1998). Divergent cAMP signal pathways regulate growth and pathogenesis in the rice blast fungus Magnaporthe grisea. Plant Cell 10, 1361-1373.

Calvo, A. M., Wilson, R. A., Bok, J. W. \& Keller, N. P. (2002). Relationship between secondary metabolism and fungal development. Microbiol Mol Biol Rev 66, 447-459.

Casas-Flores, S., Rios-Momberg, M., Rosales-Saavedra, T., Martinez-Hernandez, P., Olmedo-Monfil, V. \& Herrera-Estrella, A. (2006). Cross talk beween a fungal blue-light perception system and the cyclic AMP signaling pathway. Eukaryot Cell 5, 499-506.

Chet, I., Benhamou, N. \& Haran, S. (1998). Mycoparasitism and lytic enzymes. In Trichoderma and Gliocladium, vol. 2, Enzymes, Biological Control and Commercial Applications, pp. 153-171. Edited by G. E. Harman \& C. P. Kubicek. London: Taylor \& Francis.

Choi, W. \& Dean, R. A. (1997). The adenylate cyclase gene MAC1 of Magnaporthe grisea controls appressorium formation and other aspects of growth and development. Plant Cell 9, 1973-1983.

Fillinger, S., Chaveroche, M. K., Shimizu, K., Keller, N. \& d'Enfert, C. (2002). cAMP and ras signaling independently control spore germination in the filamentous fungus Aspergillus nidulans.. Mol Microbiol 44, 1001-1016.

Harman, G. E. \& Bjorkmann, T. (1998). Potential and existing uses of Trichoderma and Gliocladium for plant disease control and plant growth enhancement. In Trichoderma and Gliocladium, vol. 2, Enzymes, Biological Control and Commercial Applications, pp. 229265. Edited by G. E. Harman \& C. P. Kubicek. London: Taylor \& Francis.

Harman, G. E., Howell, C. R., Viterbo, A., Chet, I. \& Lorito, M. (2004). Trichoderma species - opportunistic, avirulent plant symbionts. Nat Rev Microbiol 2, 43-56.

Hicks, J. K., Yu, J.-H., Keller, N. P. \& Adams, T. H. (1997). Aspergillus sporulation and mycotoxin production both require inactivation of the FadA $\mathrm{G}_{\alpha}$ protein-dependent signaling pathway. EMBO J 16, 49164923.

Hjeljord, L. \& Tronsmo, A. (1998). Trichoderma and Gliocladium in biological control: an overview. In Trichoderma and Gliocladium, vol. 2, Enzymes, Biological Control and Commercial Applications, pp. 129-155. Edited by G. E. Harman \& C. P. Kubicek. London: Taylor \& Francis.

Howell, C. R. (1987). Relevance of mycoparasitism in the biological control of Rhizoctonia solani by Gliocladium virens. Phytopathology 77, 992-994.

Ivey, F. D., Yang, Q. \& Borkovich, K. A. (1999). Positive regulation of adenylyl cyclase activity by a $\mathrm{G}_{\alpha \mathrm{i}}$ homologue in Neurospora crassa. Fungal Genet Biol 26, 48-61.

Ivey, F. D., Kays, A. M. \& Borkovich, K. A. (2002). Shared and independent roles for a $\mathrm{G}_{\alpha \mathrm{i}}$ protein and adenylyl cyclase in regulating development and stress responses in Neurospora crassa. Eukaryot Cell $1,634-642$.

Keller, N. P., Turner, G. \& Bennett, J. W. (2005). Fungal secondary metabolism - from biochemistry to genomics. Nat Rev Microbiol 3, 937-947.

Klimpel, A., Gronover, C. S., Williamson, B., Stewart, J. A. \& Tudzynski, B. (2002). The adenylate cyclase (BAC) in Botrytis cinerea is required for full pathogenicity. Mol Plant Pathol 3, 439-450. 
Kuhls, K., Lieckfeldt, E., Börner, T. \& Gueho, E. (1999). Molecular reidentification of human pathogenic Trichoderma isolates as Trichoderma longibrachiatum and Trichoderma citrinoviride. Med Mycol 37, 25-33.

Lengeler, K. B., Davidson, R. C., D'Souza, C., Harashima, T., Shen, W. C., Wang, P., Pan, X., Waugh, M. \& Heitman, J. (2000). Signal transduction cascades regulating fungal development and virulence. Microbiol Mol Biol Rev 64, 746-785.

Liebmann, B., Gattung, S., Jahn, B. \& Brakhage, A. A. (2003). cAMP signaling in Aspergillus fumigatus in involved in the regulation of the virulence gene $p k s P$ and in defense against the killing by macrophages. Mol Genet Genomics 269, 420-435.

Liu, S. \& Dean, R. (1997). G protein alpha subunit genes control growth, development and pathogenicity of Magnaporthe grisea. Mol Plant Microbe Interact 10, 1075-1086.

Mach, R. L. \& Zeilinger, S. (2003). Regulation of gene expression in industrial fungi: Trichoderma. Appl Microbiol Biotechnol 60, 515-522.

Mukherjee, P. K., Mukhopadhyay, A. N., Sarmah, D. K. \& Shreshtha, S. M. (1995). Comparative antagonistic properties of Gliocladium virens and Trichoderma harzianum on Sclerotium rolfsii and Rhizoctonia solani - its relevance to understanding the mechanisms of biocontrol. J Phytopathol 143, 275-279.

Mukherjee, M., Hadar, R., Mukherjee, P. K. \& Horwitz, B. A. (2003a). Homologous expression of a mutated beta-tubulin gene does not confer benomyl resistance on Trichoderma virens. J Appl Microbiol 95, 861-867.

Mukherjee, P. K., Latha, J., Hadar, R. \& Horwitz, B. A. (2003b). TmkA, a mitogen activated protein kinase of Trichoderma virens, is involved in biocontrol properties and repression of conidiation in the dark. Eukaryot Cell 2, 446-455.

Mukherjee, P. K., Latha, J., Hadar, R. \& Horwitz, B. A. (2004). Role of two G-protein alpha subunits, TgaA and TgaB, in the antagonism of Trichoderma virens against plant pathogens. Appl Environ Microbiol 70, 542-549.

Mukherjee, M., Horwitz, B. A., Sherkhane, P. D., Hadar, R. \& Mukherjee, P. K. (2006a). A secondary metabolite biosynthesis cluster in Trichoderma virens: evidence from analysis of genes underexpressed in a mutant defective in morphogenesis and antibiotic production. Curr Genet 50, 193-202.

Mukherjee, P. K., Hadar, R., Pardovitz-Kedmi, E., Trushina, N. \& Horwitz, B. A. (2006b). MRSP1, encoding a novel Trichoderma secreted protein, is negatively regulated by MAPK. Biochem Biophys Res Commun 350, 716-722.

Nishimura, M., Park, G. \& Xu, J.-R. (2003). The G-beta subunit MGB1 is involved in regulating multiple steps of infection-related morphogenesis in Magnaporthe grisea. Mol Microbiol 50, 231-243.

Pastan, I. \& Perlman, R. (1970). Cyclic adenosine monophosphate in bacteria. Science 169, 339-344.
Reithner, B., Brunner, K., Schumacher, R., Peissl, I., Seidl, V., Krska, R. \& Zeilinger, S. (2005). The $G$ protein $\alpha$ subunit Tgal of Trichoderma atroviride is involved in chitinase formation and differential production of antifungal metabolites. Fungal Genet Biol 42, 749-760.

Robinson, G. A. \& Sutherland, E. W. (1971). Cyclic AMP and the function of eukaryotic cells: an introduction. Ann N Y Acad Sci 185, 5-9.

Rocha-Ramirez, V., Omero, C., Chet, I., Horwitz, B. A. \& HerreraEstrella, A. (2002). Trichoderma atroviride G-protein $\alpha$-subunit gene tgal is involved in mycoparasitic coiling and conidiation. Eukaryot Cell 1, 594-605.

Rosenberg, G. \& Pall, M. L. (1979). Properties of two cyclic nucleotide-deficient mutants of Neurospora crassa. J Bacteriol 137, $1140-1144$.

Segers, G. C. \& Nuss, D. L. (2003). Constitutively activated $G_{\alpha}$ negatively regulates virulence, reproduction and hydrophobin gene expression in the chestnut blight fungus Cryphonectria parasitica. Fungal Genet Biol 38, 198-208.

Shimizu, K. \& Keller, N. P. (2001). Genetic involvement of cAMPdependent protein kinase in a $G$ protein signaling pathway regulating morphological and chemical transitions in Aspergillus nidulans. Genetics 157, 591-600.

Sivasithamparam, K. \& Ghisalberti, E. (1998). Secondary metabolism. In Trichoderma and Gliocladium, vol. 1, Basic Biology, Taxonomy and Genetics, pp. 139-191. Edited by G. E. Harman \& C. P. Kubicek. London: Taylor \& Francis.

Terenzi, H. F., Flawia, M. M. \& Torres, H. N. (1974). A Neurospora crassa morphological mutant showing reduced adenylate cyclase activity. Biochem Biophys Res Commun 58, 990-996.

Terenzi, H. F., Flawia, M. M., Tellez-Inon, M. T. \& Torres, H. N. (1976). Control of Neurospora crassa morphology by cyclic adenosine $3^{\prime}, 5^{\prime}-$ monophosphate and dibutyryl cyclic adenosine $3^{\prime}, 5^{\prime}$-monophosphate. J Bacteriol 126, 91-99.

Viterbo, A., Harel, M., Horwitz, B. A., Chet, I. \& Mukherjee, P. K. (2005). Trichoderma mitogen-activated protein kinase signaling is involved in induction of plant systemic resistance. Appl Environ Microbiol 71, 6241-6246.

Xu, J. R. (2000). Map kinases in fungal pathogens. Fungal Genet Biol 31, 137-152.

Zeilinger, S. (2004). Gene disruption in Trichoderma atroviride via Agrobacterium-mediated transformation. Curr Genet 45, 54-60.

Zeilinger, S., Reithner, B., Scala, V., Piessl, I., Lorito, M. \& Mach, R. (2005). Signal transduction by Tga3, a novel G protein $\alpha$ subunit of Trichoderma atroviride. Appl Environ Microbiol 71, 1591-1597.

Edited by: H. A. B. Wösten 